DOI: http://dx.doi.org/10.12957/demetra.2014.14051

\title{
Orientações nutricionais em serviços de saúde: a percepção de idosos portadores de hipertensão e diabetes
}

\author{
Nutritional guidelines in health services: The perception of elderly patients with hypertension \\ and diabetes
}

Harriet Jane White ${ }^{1}$

Leticia Marín-León ${ }^{1}$

' Departamento de Saúde Coletiva, Faculdade de Ciências Médicas. Universidade Estadual de Campinas. Campinas-SP, Brasil.

Financiamento: Conselho Nacional de Desenvolvimento Científico e Tecnológico (Processo No 141845/2011-2).

Correspondência / Corrrespondence Harriet Jane Wh

E-mail: hiwhite@fcm.unicamp.br

\section{Resumo}

Objetivo: Descrever aspectos do atendimento nutricional e das práticas alimentares entre idosos em face das orientações nutricionais recebidas. Método: Estudo transversal realizado com idosos de ambos os sexos de Campinas-SP, utilizando questionário e as variáveis: demográficas e socioeconômicas, atendimento nutricional, avaliação da qualidade das orientações nutricionais recebidas, consumo alimentar. Os critérios de inclusão foram: ter 60 anos e mais, ter hipertensão arterial e diabetes mellitus, residir em Campinas e concordar em participar da pesquisa. Resultados: Participaram 150 idosos, sendo $66,7 \%$ do sexo feminino, com média de idade de 69,9 anos (dp $\pm 7,5)$. Os homens, mais do que as mulheres, estiveram em acompanhamento nutricional regular nos últimos12 meses (68\% e 32\%). Apesar de a grande maioria dos entrevistados relatar recebimento de orientações nutricionais, o consumo diário recomendado não era atendido para alimentos integrais (88\%), verduras (71\%), frutas (27\%); não restringiam sal (67\%) e gordura animal (65\%) e ainda $65 \%$ não cumpriam horário de refeições. Disseram ter recebido material educativo contendo orientações nutricionais: $80 \%$ dos homens e $64 \%$ das mulheres. No entanto, $50 \%$ das mulheres e $62 \%$ dos homens relataram ter dúvidas sobre as orientações recebidas. Conclusão: A baixa adoção das recomendações nutricionais aponta a necessidade de melhorar a capacitação dos profissionais de saúde, para que possam orientar de forma eficiente os pacientes quanto ao consumo alimentar. Esta constatação reforça a importância da atuação de uma equipe de saúde multiprofissional que motive os 
idosos a aderirem ao tratamento, viabilizando melhor controle das doenças crônicas e prevenção de complicações secundárias.

Palavras-chave: Recomendações Nutricionais. Educação Nutricional. Saúde do Idoso. Hábitos Alimentares. Hipertensão, Diabetes Mellitus.

\section{Abstract}

Objective: To describe some aspects of nutritional care and food practices in relation to received nutritional guidelines. Methods: Cross-sectional study with elderly of both sexes in the city of Campinas- SP, Brazil, using a questionnaire and the variables: demographic and socioeconomic, nutritional care, assessment of the quality of the received nutritional guidance and food intake. Inclusion criteria were: 60 years and older, having high blood pressure (hypertension) and diabetes mellitus, living in Campinas and agreeing to participate. Results: A sample of 150 elderly was studied, $66.7 \%$ were female with mean age 69.9 years (SD \pm 7.5 ). Men had been in regular nutritional counselling in the last 12 months more than women (68\% and 32\%). Although most elderly reported having received nutritional guidelines, daily recommended intake was not followed for whole foods (88\%), vegetables (71\%), fruit (27\%); they did not restrict salt (67\%) and animal fat (65\%) and even 65\% did not accomplish meal intervals. Educational material with nutritional guidance was received by $80 \%$ of men and $64 \%$ of women. However, $50 \%$ of women and $62 \%$ of men reported having doubts about the guidelines. Conclusion: Low compliance with nutritional recommendations indicates the need to improve health professionals training in counselling patients regarding food intake. This finding reinforces the importance of the performance of a multidisciplinary health team that motivates seniors to adhere to treatment, thus enabling better control of chronic diseases and the prevention of secondary complications.

Keywords: Recommended Dietary Allowances. Nutrition Education. Health of the Elderly. Food Habits. Hypertension. Diabetes Mellitus. 


\section{Introdução}

Ramos et al. ${ }^{1}$ foram os primeiros a destacar o envelhecimento populacional do Brasil, apontando as mudanças no perfil epidemiológico, com o aumento das doenças crônicas, e chamando a atenção para o elevado custo e cuidado permanente que elas demandam. Com o envelhecimento, as capacidades física e biológica declinam gradualmente e tornam os idosos mais vulneráveis às doenças crônicas. Segundo a Pesquisa Nacional por Amostra de Domicílios (PNAD) 2003, pouco mais de $53 \%$ da população de 65 anos e mais apresentava uma ou mais doenças crônicas, e $50 \%$ referiam três ou mais consultas médicas nos últimos 12 meses. $^{2}$

Nas análises da PNAD 2003 e 2008, o percentual de idosos portadores de diabetes mellitus (DM) e hipertensão arterial (HAS) associadas aumentou de 8,4\% para 11,3\% na faixa de 60 a 69 anos de idade; de $10,4 \%$ para $13,1 \%$ na faixa de 70 a 79 anos; e em idosos mais velhos, passou de $9 \%$ para $12,1 \%$. Os autores identificaram também maior prevalência nas mulheres e nas áreas urbanas. ${ }^{3}$

Malta et al. ${ }^{4}$ descreveram o elevado custo das doenças crônicas não transmissíveis, sendo que em 2006 os custos diretos com atendimento ambulatorial e internações, segundo estimativas do Ministério de Saúde foram de $\mathbf{R} \$ 7,5$ bilhões/ano. Ao apresentar a importância da carga das doenças crônicas não transmissíveis no Brasil, Schmidt et al. ${ }^{5}$ destacaram o crescimento de DM, HAS e excesso de peso, e atribuem seu aumento a mudanças negativas na dieta e diminuição da prática de atividade física.

Houve mudança da dieta tradicional brasileira para uma alimentação caracterizada por elevada densidade calórica, teor de açúcar, sal e gorduras saturadas, que, aliada ao sedentarismo, contribui para o desenvolvimento de doenças crônicas, como HAS e DM. ${ }^{6}$ As diretrizes da American Dietetic Association (ADA) $)^{7}$ e da Sociedade Brasileira de Diabetes $(\mathrm{SBD})^{8}$ reforçam a importância do acompanhamento e das orientações individualizadas, a fim de alcançar as metas do tratamento proposto, e consideram a alimentação e a atividade física umas das principais estratégias para o controle e tratamento do DM. As diretrizes da Sociedade Brasileira de Cardiologia (SBC), ${ }^{6}$ em 2007, mostraram a importância da dieta no controle das dislipidemias; e em 2010, a Sociedade Brasileira de Hipertensão $(\mathrm{SBH})^{9}$ relatou os benefícios da dieta mediterrânea, entre outras recomendações, para o controle da HAS.

Nas doenças crônicas, a adesão aos tratamentos nos países desenvolvidos é, em média, de 50\%; já nos países em desenvolvimento, esse percentual é inferior e depende da disponibilidade de serviços de saúde e das desigualdades de acesso à saúde. ${ }^{10}$ Estudo realizado no Canadá mostrou baixa adesão dos portadores de diabetes às recomendações nutricionais e enfatizou que a dieta deve ser individualizada, para que seja mais eficaz e os indivíduos tenham maior adesão às recomendações. ${ }^{11}$ 
O envelhecimento populacional, a demanda aumentada dos idosos por atendimentos de saúde, principalmente entre portadores de doenças crônicas, e a necessidade de prevenir as complicações justificam a realização deste estudo. O objetivo é descrever aspectos do atendimento nutricional e das práticas alimentares entre idosos em face das orientações nutricionais recebidas.

\section{Métodos}

Trata-se de estudo transversal realizado com idosos portadores de HAS e DM de ambos os sexos, de um serviço de saúde de Campinas-SP. Idosos que estavam em tratamento no ambulatório de Hospital Universitário foram entrevistados enquanto aguardavam para ser atendidos. Os critérios de inclusão foram: ter 60 anos ou mais; residir em Campinas; ter DM e HAS; e concordar em participar da pesquisa mediante assinatura do Termo de Consentimento Livre e Esclarecido.

Como instrumento de pesquisa, foi utilizado um questionário construído pelos autores, considerando as seguintes variáveis:

a) Demográficas e socioeconômicas: sexo (masculino e feminino), idade (60-69, 70-79, 80 e + ), estado conjugal (casado e outros), cor de pele (branca e outras), número de filhos vivos ( $0,1,2,3$ e mais), número de pessoas no domicílio (vive só, 1,2 e mais), escolaridade (0- 4 anos e 5 e mais), renda pessoal em salários mínimos $(\mathrm{SM})(<=1 \mathrm{SM}$ e $>=2 \mathrm{SM})$, aposentado (sim e não).

b) Atendimento nutricional em serviço de saúde: quanto ao atendimento nutricional, foi perguntado - "O senhor(a) fez acompanhamento com nutricionista nos últimos 3 meses?"; "O senhor(a) fez acompanhamento nutricional regular, no último ano? / ou alguma vez na vida?"

c) Orientação nutricional e práticas alimentares: Foram feitas perguntas em relação à alimentação - "O senhor(a) recebeu alguma orientação por escrito em relação à alimentação?" (dieta, lista de alimentos, ambas e nenhuma); "O senhor(a) considera que as dúvidas sobre alimentação foram esclarecidas?" (sim e não); "O senhor(a) sentiu dificuldade para seguir as orientações recebidas?" (falta de vontade, não tem dinheiro, esquece de comer nos horários, falta de conhecimentos e não recebeu orientação).

As orientações recebidas foram classificadas em "sim/não" quando o idoso referia ter ou não recebido determinada orientação. As questões sobre orientações nutricionais recebidas e as práticas alimentares em face dessas orientações foram cotejadas com as recomendações propostas pela $\mathrm{SBC}^{6}, \mathrm{SBH}^{9}, \mathrm{SBD}^{8,12}$ e $\mathrm{ADA},{ }^{7}$ sobre o consumo de: leite desnatado, verduras, frutas, leguminosas, alimentos integrais, água, sal, temperos industrializados e alimentos ricos em gorduras. 
O Guia Alimentar para a População Brasileira ${ }^{13}$ também foi utilizado como referência para o consumo e frequência alimentar. Ainda não há questionário validado exclusivamente para os idosos; assim, utilizou-se como referência um questionário para adultos. ${ }^{14} \mathrm{Em}$ relação à frequência alimentar de alimentos saudáveis, as variáveis pesquisadas e categorias de respostas foram: leite desnatado ou semidesnatado, verduras, frutas e leguminosas, alimentos integrais $(<3$ vezes na semana; 3 a 6 vezes na semana e diário); consumo de água ( $<2$ copos; 5 a 8 copos; 9 e + ). Entre os alimentos nos quais deve ser orientada a restrição, incluíram-se: sal e tempero industrializado (não/sim) e alimentos ricos em gorduras (>2 vezes na semana; 1 a 2 vezes na semana e raramente). Ainda foi indagado o horário das refeições (come o tempo todo; fica mais de 5 horas sem comer; come de 3/3 horas) e a diminuição ou manutenção do peso (não/sim).

Considerou-se consumo alimentar adequado a última categoria de respostas acima mencionadas; e foi considerado cumprimento das recomendações nutricionais a última categoria de resposta. A opção pela frequência de consumo diário de alimentos saudáveis como padrão, e não a usada pelo Vigitel ${ }^{15}$ (5 vezes ou mais), muito usada atualmente em estudos epidemiológicos, tem por base a necessidade de utilizar o melhor padrão de dieta, visto que essas recomendações fazem parte do tratamento não medicamentoso.

Na análise dos dados, o programa Epidata versão 3.1 foi utilizado para digitar o banco de dados e realizar as análises. Foram feitas análises estatísticas descritivas (frequências simples e relativas) quanto a sexo, orientações nutricionais e cumprimento de recomendações nutricionais. $\mathrm{O}$ teste de chi quadrado foi usado para verificar associação.

O projeto foi encaminhado ao Comitê de ética da Faculdade de Ciências Médicas da Universidade Estadual de Campinas e aprovado através do parecer número 115/233 (04/10/2012).

\section{Resultados}

Dos 150 idosos, 66,7\% eram mulheres; a idade dos participantes variou de 60 a 92 anos, com média de 69,9 anos $(\mathrm{dp} \pm 7,5)$. As variáveis com diferenças significativas entre homens e mulheres foram: estado conjugal, renda, escolaridade e aposentadoria, sendo maior o percentual de homens casados, com renda de 2SM ou mais, com escolaridade de cinco anos de estudo ou mais e que recebiam aposentadoria. Em geral, houve predomínio em ambos os sexos de idosos casados, de cor de pele branca, com três ou mais filhos vivos e baixa escolaridade (tabela 1). 
Tabela 1. Perfil sociodemográfico de idosos portadores de diabetes e hipertensão usuários de um ambulatório hospitalar segundo sexo (n=150). Campinas-SP, 2013.

\begin{tabular}{|c|c|c|c|c|}
\hline Variáveis & $\mathrm{N}$ & $\begin{array}{l}\text { Feminino } \\
\mathrm{n}=100(\%)\end{array}$ & $\begin{array}{c}\text { Masculino } \\
\mathrm{n}=50(\%)\end{array}$ & $\begin{array}{c}\text { Valor de } \\
\mathrm{p}^{\mathrm{a}}\end{array}$ \\
\hline Idade & & & & ns \\
\hline $60-69$ & 77 & 54,0 & 46,0 & \\
\hline $70-79$ & 54 & 33,0 & 42,0 & \\
\hline $80 \mathrm{e}+$ & 19 & 13,0 & 12,0 & \\
\hline Estado conjugal & & & & 0,030 \\
\hline Casado & 95 & 55,0 & 80,0 & \\
\hline Outro & 55 & 45,0 & 20,0 & \\
\hline Cor de pele & & & & $\mathrm{ns}$ \\
\hline Branca & 99 & 67,0 & 64,0 & \\
\hline Outras & 51 & 33,0 & 36,0 & \\
\hline Filhos vivos & & & & ns \\
\hline 0 & 9 & 5,0 & 8,0 & \\
\hline $1-2$ & 56 & 38,0 & 36,0 & \\
\hline $3 \mathrm{e}+$ & 85 & 57,0 & 56,0 & \\
\hline Número de pessoas no domicílio & & & & $\mathrm{ns}$ \\
\hline 0 & 22 & 18,0 & 8,0 & \\
\hline 1 & 67 & 43,0 & 48,0 & \\
\hline $2 \mathrm{e}+$ & 61 & 39,0 & 44,0 & \\
\hline Anos estudo & & & & 0,020 \\
\hline $0-4$ & 106 & 77,0 & 58,0 & \\
\hline $5 \mathrm{e}+$ & 44 & 23,0 & 42,0 & \\
\hline Valor rendimento & & & & 0,000 \\
\hline$<=1 \mathrm{SM}$ & 85 & 68,0 & 34,0 & \\
\hline$>=2 \mathrm{SM}$ & 65 & 32,0 & 66,0 & \\
\hline Aposentado & & & & 0,000 \\
\hline Sim & 88 & 46,0 & 84,0 & \\
\hline Não & 62 & 54,0 & 16,0 & \\
\hline
\end{tabular}

avalor de $\mathrm{p}$ do teste de chi-quadrado; $\mathrm{SM}=$ salário mínimo.

$\mathrm{ns}=$ não significativo $\mathrm{p}>=0,05$ 
Embora sem diferença estatisticamente significativa, os homens referiram maior frequência de consulta com nutricionista que as mulheres, tanto nos últimos três meses (40\% e 28\%) quanto nos últimos 12 meses (68\% e 32\%), e alguma vez na vida (90\% e 74\%), segundo a tabela 2.

Tabela 2. Frequência de respostas relativas ao atendimento em serviços de saúde segundo tipo de profissional consultado e sexo do paciente portador de diabetes e hipertensão(n=150). Campinas-SP, 2013.

\begin{tabular}{|c|c|c|c|c|}
\hline Variáveis & $\mathrm{N}$ & $\begin{array}{c}\text { Feminino }=100 \\
(\%)\end{array}$ & $\begin{array}{l}\text { Masculino } \\
\mathrm{n}=50(\%)\end{array}$ & $\begin{array}{l}\text { Valor } \\
\text { de } \mathrm{p}^{\mathrm{a}}\end{array}$ \\
\hline $\begin{array}{l}\text { Atendimento com nutricionista } \\
\text { nos últimos } 3 \text { meses }\end{array}$ & & & & ns \\
\hline Sim & 48 & 28,0 & 40,0 & \\
\hline $\begin{array}{l}\text { Acompanhamento nutricional } \\
\text { regular nos últimos } 12 \text { meses }\end{array}$ & & & & 0,023 \\
\hline Sim & 55 & 32,0 & 68,0 & \\
\hline $\begin{array}{l}\text { Acompanhamento nutricional } \\
\text { alguma vez na vida }\end{array}$ & & & & ns \\
\hline Sim & 119 & 74,0 & 90,0 & \\
\hline $\begin{array}{l}\text { Tipo orientação escrita sobre } \\
\text { alimentação }\end{array}$ & & & & ns \\
\hline Dieta e lista de alimentos & 12 & 7,0 & 10,0 & \\
\hline Lista de alimentos & 92 & 57,0 & 70,0 & \\
\hline Nenhuma & 46 & 36,0 & 20,0 & \\
\hline Dúvidas sobre alimentação & & & & ns \\
\hline Sim & 81 & 50,0 & 62,0 & \\
\hline $\begin{array}{l}\text { Dificuldades para seguir as } \\
\text { orientações recebidas }^{b}\end{array}$ & & & & ns \\
\hline Falta de vontade & 74 & 49,0 & 50,0 & \\
\hline Não tem dinheiro & 39 & 29,0 & 20,0 & \\
\hline Esquece comer nos horários & 69 & 49,0 & 42,0 & \\
\hline Falta de conhecimento & 30 & 18,0 & 24,0 & \\
\hline Não recebeu orientação & 35 & 25,0 & 20,0 & \\
\hline
\end{tabular}

${ }^{a} \mathrm{p}$ valor do teste de chi-quadrado

$\mathrm{ns}=$ não significativo $\mathrm{p}>=0,05$

${ }^{\mathrm{b}}$ As pessoas podiam responder mais de uma alternativa, por isso o total ultrapassa o $100 \%$. 
No presente estudo, $80 \%$ e $64 \%$ dos homens e das mulheres, respectivamente, afirmaram ter recebido algum tipo de material educativo por escrito sobre orientações nutricionais. É alta a frequência de dúvidas em relação às orientações (62\% dos homens e 50\% das mulheres). E ainda, $49 \%$ das mulheres e $50 \%$ dos homens relataram falta de vontade para comer o que foi recomendado, e 49\% das mulheres e 42\% dos homens não comem nos horários recomendados (tabela 2).

Em relação às orientações nutricionais, todos os idosos relataram recebimento de orientações relacionadas à necessidade de consumo de verduras, frutas e água; no entanto, as orientações não são seguidas por uma parcela dos entrevistados. Somente $28,7 \%$ consumiam verduras diariamente, e a grande maioria $(91,4 \%)$ consumia apenas uma porção por refeição (dados não apresentados). Quanto às frutas, 73,3\% consumiam diariamente, porém apenas uma porção. Somente 13,3\% bebiam menos de cinco copos de agua por dia (tabela 3).

Em relação ao consumo de leite desnatado, 93,3\% receberam orientação e 74,3\% da amostra relataram consumo diário, mas apenas de um copo/dia. Embora quase $40 \%$ não tenham recebido orientação sobre a necessidade de consumo de leguminosas, os percentuais em todas as categorias são muito semelhantes aos que receberam orientação, atingindo quase $90 \%$ nas categorias de maior consumo. Apenas em relação aos alimentos integrais foi observado consumo significativamente maior nos idosos que receberam a orientação (12,1\% e 2\% de consumo diário), segundo a tabela 3 .

A parcela que não cumpria com as orientações de consumo diário variou segundo o alimento: alimentos integrais $(87,9 \%)$, leguminosas $(78,3 \%)$, verduras $(71,3 \%)$, frutas $(26,7 \%)$ e leite desnatado ou semidesnatado (25,7\%). Todos os idosos referiram ter recebido orientação para diminuir o consumo de sal, mas somente 33,3\% relataram restringi-lo. Já a orientação para restringir o consumo de tempero pronto foi recebida por $41,3 \%$ dos indivíduos, e quanto ao cumprimento desta restrição não houve diferença em relação aos que não receberam orientação (59\% e 58\%). A restrição do consumo de alimentos ricos em gordura animal atingiu 92,7\%, mas seu consumo semanal foi alto (63,6\% nos que não receberam orientação e $59 \%$ nos que foram orientados). Assim, a porcentagem de não cumprimento das restrições foi de $66,7 \%$ para sal, $41 \%$ para temperos industriais e $69 \%$ para gordura animal.

A respeito da importância do horário das refeições, $88,7 \%$ declararam ter recebido esta orientação, sendo que apenas $34,6 \%$ a seguem adequadamente; $52,6 \%$ ficam mais de cinco horas sem comer e 12,8\% comem o tempo todo - portanto $65,4 \%$ não cumprem a recomendação de horário das refeições (tabela 3).

Quanto às orientações sobre a manutenção ou perda de peso, $82 \%$ receberam esta orientação, mas o percentual que ganhou peso foi um pouco superior nos que foram orientados (26\% e 22,2\%), segundo a tabela 3 . 
Tabela 3. Frequência de recebimento de orientações nutricionais / atividade física e seguimento das recomendações segundo frequência de consumo ou do comportamento, em portadores de diabetes e hipertensão $(n=150)$. Campinas-SP, 2013

\begin{tabular}{|c|c|c|c|c|}
\hline \multirow[t]{2}{*}{$\begin{array}{l}\text { Tipo deorientação } \\
\text { nutricional }\end{array}$} & \multirow{2}{*}{$\begin{array}{c}\text { Recebeu } \\
\text { orientação } \\
(\%)\end{array}$} & \multicolumn{3}{|c|}{$\begin{array}{c}\text { Seguimento das recomendações } \\
\text { nutricionais }(\%)\end{array}$} \\
\hline & & $<3$ vezes/sem & 3-6 vezes/sem & Diário \\
\hline \multicolumn{5}{|c|}{ Leite desnatado ou semi } \\
\hline Sim & 93,3 & 5,0 & 20,7 & $74,3^{\mathrm{a}}$ \\
\hline Não & 6,7 & 10,0 & 30,0 & 60,0 \\
\hline \multicolumn{5}{|l|}{ Verduras } \\
\hline Sim & 100 & 35,3 & 36,0 & $28,7^{\mathbf{b}}$ \\
\hline \multicolumn{5}{|l|}{ Frutas } \\
\hline $\operatorname{Sim}$ & 100 & 5,4 & 21,3 & $73,3^{\mathbf{c}}$ \\
\hline \multicolumn{5}{|l|}{ Leguminosas } \\
\hline $\operatorname{Sim}$ & 61,3 & 13,0 & 65,3 & 21,7 \\
\hline Não & 38,7 & 13,8 & 65,5 & 20,7 \\
\hline \multicolumn{5}{|l|}{ Alimentos integrais } \\
\hline Sim & 66,0 & 60,6 & 27,3 & 12,1 \\
\hline Não & 34,0 & 90,2 & 7,8 & 2,0 \\
\hline Água & & $<5 \operatorname{copos}^{\mathrm{d}}$ & $5-8 \operatorname{copos}^{\mathrm{d}}$ & $9 e+\operatorname{copos}^{d}$ \\
\hline Sim & 100 & 13,3 & 18,7 & 68,0 \\
\hline \multicolumn{5}{|l|}{ RESTRITOS } \\
\hline Sal & & Livre & & Restrito \\
\hline Sim & 100 & 66,7 & & 33,3 \\
\hline Não & 0 & 0 & & 0 \\
\hline Temperos prontos & & Livre & & Restrito \\
\hline Sim & 41,3 & 41,0 & & 59,0 \\
\hline Não & 58,7 & 42,0 & & 58,0 \\
\hline $\begin{array}{l}\text { Alimentos ricos em } \\
\text { gordura }\end{array}$ & & $\begin{array}{l}\text { >2 vezes/ } \\
\text { sem }\end{array}$ & $\begin{array}{l}\text { 1-2 vezes/ } \\
\text { sem }\end{array}$ & Raramente \\
\hline Sim & 92,7 & 7,2 & 51,8 & 41,0 \\
\hline Não & 7,3 & 0 & 63,6 & 36,4 \\
\hline
\end{tabular}




\begin{tabular}{ll|ccc}
\hline OUTRAS ORIENTAÇÕES & & & \\
\hline $\begin{array}{l}\text { Seguir o horário das } \\
\text { refeições }\end{array}$ & & $\begin{array}{c}\text { Come o } \\
\text { tempo todo }\end{array}$ & $\begin{array}{c}\text { Mais de 5 } \\
\text { horas sem } \\
\text { comer }\end{array}$ & $\begin{array}{c}\text { Come de } \\
\mathbf{3} / \mathbf{3} \text { horas }\end{array}$ \\
Sim & 88,7 & 12,8 & 52,6 & 34,6 \\
Não & 11,3 & 17,7 & 64,7 & 17,6 \\
\hline Manter/ Perder peso & & Ganhou & Manteve & Perdeu \\
Sim & 82,0 & 26,0 & 50,4 & 23,6 \\
Não & 18,0 & 22,2 & 51,9 & 25,9 \\
\hline
\end{tabular}

aPorção equivalente a uma xícara

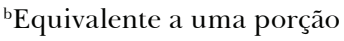

${ }^{c}$ Unidade ou uma fatia de fruta

${ }^{\mathrm{d}}$ Copos por dia

\section{Discussão}

O perfil demográfico dos idosos deste estudo se assemelha ao de inquéritos populacionais de saúde, com predomínio de mulheres, idosos na faixa etária de 60 a 69 anos e indivíduos com baixa renda e escolaridade. ${ }^{2,16}$

Há considerações a fazer quanto a limitações e pontos positivos do estudo. O tamanho da amostra não permitiu que fossem observadas diferenças significativas de adoção das recomendações nutricionais segundo ter ou não recebido orientação. A população estudada, embora atendida no ambulatório de um único hospital, é de certa forma representativa da cidade, visto que o local de moradia dos sujeitos está disperso pelos diversos distritos e áreas de abrangência dos serviços de saúde. O estudo realizado com base na percepção dos idosos ajuda na compreensão das práticas educativas pelo prisma de quem a vivencia, e permite também avaliar as expectativas dos pacientes em relação ao modo como os profissionais dos serviços de saúde abordam as necessidades dos idosos.

Este estudo é consistente com a pesquisa de Guimarães \& Takayanagui, ${ }_{17}^{17}$ sobre aconselhamentos nutricionais em pacientes diabéticos de Ribeirão Preto. Os autores observaram que a maioria das orientações era prescrita por médicos (96,5\%), mas apenas 17,2\% seguiam as recomendações sobre dieta.

No presente estudo, o cumprimento da orientação nutricional de manter ou diminuir peso foi de $74 \%$. Resultados similares aos nossos foram encontrados por Assunção et al., ${ }^{18}$ em estudo com 378 diabéticos realizado em Pelotas, quanto a perda ou manutenção de peso, no qual $72 \%$ dos idosos receberam orientação e apenas $26,5 \%$ cumpriram a recomendação. No presente estudo, porcentagem 
semelhante (80\%) recebeu essa orientação, mas o cumprimento foi maior (45\%). Ainda comparando este estudo com o de Assunção et al., ${ }^{18}$ em relação à orientação nutricional, o percentual que recebeu orientação foi semelhante (80\% e 85\%), porém o cumprimento neste foi maior (74\% e 53\%).

No estudo de Paiva et al. ${ }^{19}$ com 72 pacientes portadores de hipertensão ou diabetes realizado em Francisco Morato-SP, foi menor o percentual de idosos que declararam ter recebido orientações sobre manutenção do peso (59,4\%); no entanto, 75,0\% dos idosos seguiam a dieta de forma adequada ou parcial.

Estudo com amostra aleatória, realizado com 785 hipertensos e 823 diabéticos atendidos em 208 equipes da Estratégia Saúde da Família, distribuídas em 35 municípios do estado de Pernambuco$\mathrm{PB}$, aponta que, embora mais de $70 \%$ de cada grupo apresentasse excesso de peso, somente $16 \%$ dos hipertensos e $14 \%$ dos diabéticos referiram seguir a recomendação para perda de peso. $\mathrm{O}$ cumprimento da restrição de sal foi elevado (90\%), sendo maior nos municípios de pequeno porte e sobretudo nos diabéticos com hipertensão associada. ${ }^{20}$

O cumprimento das orientações de dieta, no presente estudo, é superior ao observado na década de 1990 em pesquisa de abrangência nacional com adultos hipertensos, diabéticos, obesos e com hiperlipidemia, realizada nos EUA. Essa observou cumprimento da dieta em menos de $45 \%$ dos indivíduos, percentual que foi mais elevado nos sujeitos que apresentaram três fatores de risco cardiovascular, nos quais o cumprimento foi de $59 \% .{ }^{21}$ Portanto, a aderência às recomendações nutricionais nesse estudo, em sujeitos em condição clínica semelhante aos de nossa amostra, foi inferior aos nossos sujeitos.

No presente estudo, embora a grande maioria dos entrevistados tenha declarado receber orientações nutricionais, os idosos consumiam menos do que o recomendado, de acordo com as diretrizes da $\mathrm{ADA},{ }^{7} \mathrm{SBD},{ }^{8,12} \mathrm{SBC}^{6}$ e $\mathrm{SBH},{ }^{9}$ em relação a: verduras, frutas, legumes e alimentos integrais, sendo que grande parte não as consumia diariamente. Em relação ao comportamento alimentar não saudável, observou-se elevada ingestão de sal, temperos prontos e gorduras animais, e apenas uma pequena parcela mantinha os intervalos adequados entre as refeições.

A importância da alimentação saudável para a prevenção primária de síndrome metabólica é destacada por estudo de coorte francês, que aponta que os indivíduos que seguiam as recomendações nutricionais apresentaram menor chance de desenvolver sindrome metabólica, havendo sinergismo entre dieta e atividade fisica. ${ }^{22}$

Apesar de os idosos receberem orientações nutricionais, grande parte não as seguia, provavelmente por não entender as orientações recebidas e/ou se sentir desmotivado para cumprilas e não compreender a importância das medidas para o controle das doenças. Para Cotta et al., ${ }^{23}$ o desconhecimento sobre a natureza multifatorial do DM e da HAS dificulta a compreensão do tratamento e diminui o cumprimento. Concordamos com os autores, quanto à necessidade de 
estimular o empoderamento dos pacientes, mediante educação em grupos que possa permitir autonomia nas escolhas, decisões e comportamentos frente ao tratamento.

Na Pesquisa de Orçamentos Familiares - POF 2008-2009, ${ }^{24}$ constatou-se consumo insuficiente de frutas, verduras e legumes, além de consumo excessivo de gorduras de origem animal. Há semelhança entre o comportamento alimentar da população geral e o comportamento dos idosos portadores de HAS e DM do presente estudo. Esta constatação evidencia a complexidade do controle das doenças e a necessidade deconsiderar os determinantes econômicos, culturais e ambientais que possam influenciar nas escolhas alimentares. Estudo de revisão sistemática referenciou vários estudos internacionais em que se observou maior dificuldade para realizar escolhas saudáveis em população de baixa renda. ${ }^{25}$

Considerando os resultados de baixo cumprimento das recomendações nutricionais e insuficiente orientação observados no presente estudo e em diversos outros, fica evidente a necessidade de melhorar a qualidade do atendimento, não só investigando fatores individuais e relacionados à qualidade dos serviços de saúde, mas também abordando-os de maneira global, incluindo os aspectos sociais e culturais, utilizando estratégias de ação voltadas para a população, sobretudo a mais vulnerável.

No estudo de Assunção \& Ursine ${ }^{26}$ realizado com 164 pacientes portadores de diabetes de um centro de saúde de Belo Horizonte-MG, os autores descreveram elevada adesão às orientações não medicamentosas. Esse comportamento é explicado pelo fato de os indivíduos participarem de grupos específicos para diabéticos e se sentirem motivados com o tratamento. Desta forma, fica clara a motivação como uma das estratégias para melhorar a compreensão das doenças, pois a adesão ao tratamento e a participação em grupo ampliam a rede de apoio social e permitem um aprendizado dinâmico na interação com os outros participantes.

Ainda nesta abordagem, considera-se importante apresentar o novo Guia Alimentar para a População Brasileira ${ }^{27}$ e discutir o valor nutritivo dos alimentos ultraprocessados, incentivar o consumo de alimentos saudáveis e valorizar os produtos in natura e o preparo de comidas caseiras. Santos $^{28}$ destaca a necessidade de uma educação fundamentada na promoção de qualidade de vida e saúde, e não baseada no medo da morte.

Diante do exposto, mostra-se necessário melhorar a capacitação dos profissionais de saúde para orientar os pacientes portadores de diabetes e hipertensão, transcendendo apenas o tratamento medicamentoso.

\section{Conclusão}

O baixo cumprimento das recomendações nutricionais de pacientes portadores de HAS e DM aponta para a necessidade de melhorar a capacitação dos profissionais de saúde para motivar os 
pacientes quanto à alimentação. Reforça também a importância da atuação de uma equipe de saúde multiprofissional, de modo a viabilizar o melhor controle das doenças crônicas e a prevenção de complicações secundárias.

\section{Referências}

1. Ramos LR, Veras RP, Kalache A. Envelhecimento populacional: uma realidade brasileira. Rev. Saúde Pública 1987; 21:211-24.

2. Lima-Costa MF, Matos DL, Camarano AA. Health inequality trends among Brazilian adults and old-aged: a study based on the National Household Sample Survey (PNAD 1998, 2003). Cienc. Saúde Coletiva 2006; 11(4):941-50.

3. Freitas LRS, Garcia LP. Evolução da prevalência do diabetes e deste associado à hipertensão arterial no Brasil: análise da Pesquisa Nacional por Amostra de Domicílios, 1998, 2003 e 2008. Epidemiol. Serv. Saúde 2012; 21(1):7-19.

4. Malta DC, Cezário AC, Moura L, Morais Neto OL, Silva Junior JB. A construção da vigilância e prevenção das doenças crônicas não transmissíveis no contexto do Sistema Único de Saúde. Epidemiol. Serv. Saúde 2006; 15(3):47-65.

5. Schmidt MI, Duncan BB, Azevedo e Silva G, Menezes AM, Monteiro CA, Barreto SM, et al. Chronic non-communicable diseases in Brazil: burden and current challenges. Lancet 2011; 377(9781):1949-61.

6. Sociedade Brasileira de Cardiologia. IV diretriz brasileira sobre dislipidemias e prevenção da Aterosclerose Departamento de Aterosclerose da Sociedade Brasileira de Cardiologia. Arquivos Brasileiros de Cardiologia 2007; 88(Supl. I):1-19.

7. American Diabetes Association. Standards of medical care in Diabetes. Diabetes Care 2014; 37 (Supp11): S14-S580.

8. Sociedade Brasileira de Diabetes. Diretrizes da Sociedade Brasileira de Diabetes. Rio de Janeiro: SBD; 2009.

9. Sociedade Brasileira de Hipertensão. VI diretrizes brasileiras de hipertensão. Capitulo 5. Tratamento não medicamentoso e abordagem multiprofissional. Rev. Bras. Hipertens 2010; 17(1):16-20.

10. World Health Organization. Hypertension. In: World Health Organization. Adherence to long-term therapies. Evidence for action. Geneva: WHO; 2003. p. 107-114).

11. Sievenpiper JL, Dworatzek PD. Food and dietary pattern-based recommendations: an emerging approach to clinical practice guidelines for nutrition therapy in diabetes. Can. J. Diabetes 2013, 37(1):51-57.

12. Sociedade Brasileira de Diabetes. Algoritmo para o tratamento do diabetes tipo 2. Atualização 2011. São Paulo: SBD; 2011. Posicionamento oficial SBD n. 3.

13. Brasil. Ministério da Saúde. Guia alimentar para a população brasileira: promovendo a alimentação saudável. Brasília: Ministério da Saúde; 2008. 
14. Fisberg RM, Colucci ACA, Morimoto JM, Marchioni DML. Questionário de frequência alimentar para adultos com base em estudo populacional. Rev. Saúde Pública 2008; 42(3):550-554.

15. Brasil. Ministério da Saúde. Vigitel Brasil 2012: vigilância de fatores de risco e proteção para doenças crônicas por inquérito telefônico. Brasília: Ministério da Saúde; 2013.

16. Lebrão ML, Laurenti R. Saúde, bem-estar e envelhecimento: o estudo SABE no Município de São Paulo. Rev. Bras. Epidemiol. 2005; 8(2):127-141.

17. Guimarães FPM, Takayanagui AMM. Orientações recebidas do serviço de saúde por pacientes para o tratamento do portador de diabetes mellitus tipo 2. Rev. Nutr. 2002; 15(1):37-44.

18. Assunção MCF, Santos IS, Gigante PD. Atenção primária em diabetes no Sul do Brasil: estrutura, processo e resultado. Rev. Saúde Pública 2001; 35(1):88-95.

19. Paiva DCP, Bersusa AAS, Escuder MML. Avaliação da assistência ao paciente com diabetes e/ou hipertensão pelo Programa Saúde da Família do Município de Francisco Morato, São Paulo, Brasil. Cad. Saúde Pública 2006; 22(2):377-385.

20. Fontbonne A, Cesse EAP, Sousa IMC, Souza WV, Chaves VLV, Bezerra AFB, et al. Risk factor control in hypertensive and diabetic subjects attended by the Family Health Strategy in the State of Pernambuco, Brazil: the SERVIDIAH Study. Cad. Saúde Pública 2013; 29(6):1195-1204.

21. Ma J, Urizar Jr GG, Alehagn T, Stafford RS. Diet and physical activity counselling during ambulatory care visits in the United States. Prev. Med. 2004; 39(4):815-822.

22. Kesse-Guyot E, Fezeu L, Galan P, Hercberg S, Czernichow S, Castetbon K. Adherence to French nutritional guidelines is associated with lower risk of metabolic syndrome. J. Nutr 2011; 141(6):1134-39.

23. Cotta RMM, Reis RS, Carvalho ALB, Batista KCS, Castro FAF, Alfenas RCG. Reflexões sobre o conhecimento dos usuários no contexto do Programa de Saúde da Família: a lacuna entre o saber técnico e o popular. Physis: Rev. Saúde Coletiva 2008; 18(4):745-766.

24. Levy RB, Claro RM, Mondini L, Sichieri R, Monteiro CA. Distribuição regional e socioeconômica da disponibilidade domiciliar de alimentos no Brasil em 2008-2009. Rev. Saúde Pública 2012; 46(1):6-15.

25. Belon AP, Nykiforuk C. Possibilities and challenges for physical and social environment research in Brazil: a systematic literature review on health behaviors. Cad. Saúde Pública 2013; 29(10):1955-73.

26. Assunção TS, Ursine PGS. Estudo de fatores associados à adesão ao tratamento não farmacológico em portadores de diabetes mellitus assistidos pelo Programa Saúde da Família, Ventosa, Belo Horizonte. Cienc. Saúde Coletiva 2008; 13(Sup. 2):2189-97.

27. Brasil. Ministério da Saúde. Guia alimentar para a população brasileira 2. ed. Brasília: Ministério da Saúde; 2014.

28. Santos LAS. O fazer educação alimentar e nutricional: algumas contribuições para reflexão. Cienc. Saúde Coletiva 2012; 17(2):453-462.

Recebido: $12 / 12 / 2014$

Aprovado: 19/12/2014 